\title{
Infiltration in porous media with dynamic capillary pressure: travelling waves
}

\author{
C. CUESTA ${ }^{1}$, C. J. van DUIJN ${ }^{1}$ and J. HULSHOF ${ }^{2}$ \\ ${ }^{1}$ CWI, P.O Box 94079, 1090 GB Amsterdam, The Netherlands \\ (email: $\{$ carlota, hansd\}@cwi.nI) \\ ${ }^{2}$ Mathematical Department of Leiden University, Niels Bohrweg 1, \\ 2333 CA Leiden, The Netherlands \\ (email: hulshof@wi.leidenuniv.nl)
}

(Received 16 November 1999; revised 25 April 2000)

\begin{abstract}
We consider a model for non-static groundwater flow where the saturation-pressure relation is extended by a dynamic term. This approach, together with a convective term due to gravity, results in a pseudo-parabolic Burgers type equation. We give a rigorous study of global travelling-wave solutions, with emphasis on the role played by the dynamic term and the appearance of fronts.
\end{abstract}

\section{Introduction}

Capillary pressure is an essential characteristic of two-phase flow in porous media. It is the empirical macroscopic description of the pressure differences, due to surface tension, between the phases in the pores of an elementary representation volume.

In the standard approach, capillary pressure is expressed as a monotone function of one of the phase saturations: decreasing in terms of the wetting phase saturation and increasing in terms of the non-wetting phase saturation. Such expressions are based on static conditions within an elementary representative volume (see, for instance, Bear $[3,4]$ or Bedrikovetsky [5]).

Recently (see Gray \& Hassanisadeh [12]), new and more realistic models have been proposed to include non-static conditions as well. In its simplest form this leads to a capillary pressure, which is now a function of the wetting phase saturation and its time derivative. The formulation, with this improved capillary pressure, results in a transport equation containing higher order mixed derivatives. This equation is subject of study in this paper.

We confine ourselves to the particular case of unsaturated groundwater flow, where imbibition takes place under the influence of gravity. The two phases are water (wetting phase) and air (non-wetting phase). Throughout we assume relative small values of the water saturation, so that regions where the porous medium is fully saturated do not occur, as described by Hulshof [13] and van Duijn \& Peletier [9] for example. According to Bear $[3,4]$ for water in a homogeneous and isotropic porous medium we have the momentum balance equation

$$
\boldsymbol{q}=-K(S)\left(\nabla p_{w}+\rho g \boldsymbol{e}_{z}\right) \quad(\text { Darcy’s law })
$$


and the mass balance

$$
\phi \partial_{t}(\rho S)+\operatorname{div}(\rho \boldsymbol{q})=0 .
$$

Here $q$ denotes volumetric water flux, $S$ water saturation, $K(S)$ hydraulic conductivity, $p_{w}$ water pressure, $\rho$ water density, $\phi$ porosity and $g$ the gravity constant. Further $e_{z}$ is the unit vector in positive $z$-direction, i.e. against the direction of gravity. To solve equations (1.1) and (1.2), an additional relation between $p_{w}$ and $S$ is needed. This relation is based on the assumption that the air pressure, $p_{a}$, is constant in space and time. This assumption is common practice in unsaturated groundwater flow.

Under static conditions (see Bear [3, 4] and Bedrikovetsky [5]), one uses

$$
p_{a}-p_{w}=p_{c}(S),
$$

where $p_{c}$ denotes the monotone capillary pressure. Under non-static conditions, Gray \& Hassanisadeh [12] proposed

$$
p_{a}-p_{w}=p_{c}(S)-\phi L(S) \partial_{t} S
$$

to capture the difference between drainage and imbibition. Here $L(S)$ is a nonlinear damping term. Using homogenization techniques, Bourgeat \& Panfilov [6] derive a similar model for the capillary pressure in water-oil system containing small scale heterogeneities.

Equations (1.1), (1.2) and (1.4) can be combined into a single equation for $S$, which reads

$$
\phi \partial_{t}(\rho S)=\operatorname{div}\left\{\rho K(S) \rho g \boldsymbol{e}_{\boldsymbol{z}}+\rho K(S) \nabla\left(-p_{c}(S)+\phi L(S) \partial_{t} S\right)\right\} .
$$

Assuming now a one-dimensional flow in the vertical $z$-direction, with $\rho$ and $\phi$ constant, and applying a straightforward scaling, equation (1.5) reduces to

$$
\partial_{t} S=\partial_{z}\left\{K(S)+K(S) \partial_{z}\left(-p_{c}(S)+L(S) \partial_{t} S\right)\right\} .
$$

In previous work, Barenblatt $[2,1]$ proposed a different model to describe the non-static situation. He modified (1.1) and (1.3) by replacing $S$ by $S+\tau \partial_{t} S(\tau>0)$, into $p_{c}(S)$ and $K(S)$. The resulting equation then is of the form

$$
\partial_{t} S=\partial_{z z}\left\{\Phi_{1}\left(S+\tau \partial_{t} S\right)\right\}+\partial_{z}\left\{\Phi_{2}\left(S+\tau \partial_{t} S\right)\right\},
$$

where $\Phi_{1}$ and $\Phi_{2}$ are nonlinear positive functions, and the equation is degenerate at $S+\tau \partial_{t} S=0$. This equation admits a splitting into two equations which decouple the space and time derivatives, namely,

$$
\left\{\begin{array}{l}
w=S+\tau \partial_{t} S \\
-\tau\left(\partial_{z z} \Phi_{1}(w)+\partial_{z} \Phi_{2}(w)\right)+w=S .
\end{array}\right.
$$

Thus, it can be studied as an ODE problem in Banach spaces. Moreover maximum principles are applicable [8]. There is a similar splitting for (1.6), which reads

$$
\left\{\begin{array}{l}
w=\partial_{t} S-p_{c}(S) \\
-\partial_{z}\left(K(S) \partial_{z} w\right)+w=\partial_{z} K(S)-p_{c}(S) .
\end{array}\right.
$$

The appearance of $K(S)$ as a coefficient in the $w$-equation, however, does not allow the maximum principle to be applied as in Dibenedetto \& Pierre [8]. 
We note here that if the Barenblatt ansatz $\left(S \leftrightarrow S+\tau \partial_{t} S\right)$ is applied only in (1.3), then (1.6) would result with

$$
L(S)=-\frac{\tau}{\phi} \frac{d p_{c}}{d S},
$$

implying that $L(S)$ becomes unbounded as $S \downarrow 0$. However, experiments carried out by Smiles et al. [15] (see also Hassanizadeh [11] for an overview) show that $L(S)$ vanishes as $S \downarrow 0$.

To investigate the role of the nonlinear terms (i.e. $K(S), p_{c}(S)$ and $L(S)$ ), we replace them by power-law relations. Note that this is consistent with the assumption of small water saturation. We approximate

$$
\begin{array}{rc}
K(S)=S^{\alpha} & (\alpha>1), \\
p_{c}(S)=-1+S^{-\beta} & (\beta>0), \\
L(S)=\varepsilon S^{\gamma} & (\gamma>0),
\end{array}
$$

where $\varepsilon>0$ is introduced as a parameter to investigate the consequence of the thirdorder mixed term. The parameter ranges are chosen to capture the relevant physical properties of unsaturated flow. In particular, we want $K$ and $L$ to be non-negative, with $L(0)=K(0)=K^{\prime}(0)=0$, and $p_{c}\left(0^{+}\right)=\infty$. Using these power-law relations, equation (1.6) reduces to

$$
\partial_{t} S=\partial_{z}\left\{S^{\alpha}+\beta S^{\alpha-\beta-1} \partial_{z} S+\varepsilon S^{\alpha} \partial_{z}\left(S^{\gamma} \partial_{t} S\right)\right\} .
$$

The static capillary pressure relation (1.3) would have resulted in the convection-diffusion equation

$$
\partial_{t} S=\partial_{z}\left\{S^{\alpha}+\beta S^{\alpha-\beta-1} \partial_{z} S\right\} .
$$

It is well-known (e.g. see Gilding [10]) that this equation has finite speed of propagation if and only if

$$
\int_{0}^{\delta} \frac{D(S)}{S} d S<\infty \text { for some } \delta>0 \text {, with } D(S)=\beta S^{\alpha-\beta-1} .
$$

This requires $\alpha-\beta>1$. Because the occurrence of fronts is of particular interest, we analyse equation (1.10) in the parameter range

$$
\beta, \gamma>0 \text { and } \beta<\alpha-1 \text {. }
$$

In this paper, we analyse travelling-wave solutions of (1.10). They are conjectured to describe the large time behaviour of solutions resulting from a certain class of initial conditions. The stability properties of travelling waves are the subject of a separate future study.

Thus we consider

$$
S(z, t)=f(\eta) \text { with } \quad \eta=z+c t,
$$

subject to the boundary conditions

$$
f(\infty)=A>0, \quad f(-\infty)=\delta \geqslant 0 \quad(\delta<A) .
$$

Hence, the fluid moves downwards whenever the wave speed $c$ is positive. For $f$ we obtain 
the equation

$$
c f^{\prime}=\left\{f^{\alpha}+\beta f^{\alpha-\beta-1} f^{\prime}+c \varepsilon f^{\alpha}\left(f^{\gamma} f^{\prime}\right)^{\prime}\right\}^{\prime} \text { on } \mathbb{R} .
$$

Integration and application of boundary conditions give the equation

$$
c(f-\delta)=f^{\alpha}-\delta^{\alpha}+\beta f^{\alpha-\beta-1} f^{\prime}+c \varepsilon f^{\alpha}\left(f^{\gamma} f^{\prime}\right)^{\prime},
$$

and the second-order boundary value problem

$$
(T W)\left\{\begin{array}{l}
c(f-\delta)=f^{\alpha}-\delta^{\alpha}+\beta f^{\alpha-\beta-1} f^{\prime}+c \varepsilon f^{\alpha}\left(f^{\gamma} f^{\prime}\right)^{\prime} \quad \text { on } \mathbb{R}, \\
f(-\infty)=\delta, \quad f(+\infty)=A,
\end{array}\right.
$$

where $c$ is given by

$$
c=\frac{A^{\alpha}-\delta^{\alpha}}{A-\delta}
$$

which is the Rankine-Hugoniot wave speed if we interpret (1.10) as a regularization of the hyperbolic equation $\partial_{t} S=\partial_{x} S^{\alpha}$. Note that $c \downarrow A^{\alpha-1}$ as $\delta \downarrow 0, c \uparrow \alpha A^{\alpha-1}$ as $\delta \uparrow A$, with $\frac{d c}{d \delta}>0$ for $0<\delta<A$.

In $\$ 2$ we show existence of travelling waves for fixed positive values of $\varepsilon$ and $\delta$. They are unique up to translations in $\eta$. This analysis also shows an oscillatory, but non-periodic, behaviour of the profiles. Here the value of $\varepsilon$ is crucial; for $\varepsilon$ sufficiently small (depending on $\alpha, \beta, \gamma, \delta$ and $A$ ) we obtain monotone profiles.

In $\S 3$ we study the limit case $\varepsilon \rightarrow 0$, while $\delta>0$ is kept fixed. Using monotonicity for small $\varepsilon$, we obtain convergence to travelling waves of equation (1.11), i.e. the standard model based on the static pressure saturation relation (1.3).

In $\S 4$ we analyse existence of front solutions to Problem $T W$ with $\delta=0$. It turns out that there are two relevant ranges of powers $\alpha, \beta, \gamma$ for which fronts appear. In the range $2 \beta>\alpha-\gamma-2$ there exists a family of solutions which degenerate at a finite value of

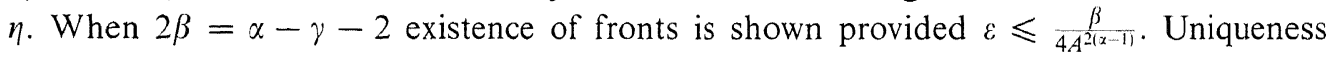
does not hold. Nevertheless, we have discerned in each of the previous cases a unique (up to translations in $\eta$ ) exceptional profile, which is the limit profile to $(T W)$ when letting $\delta \rightarrow 0$. This is shown in $\S 5$. In the other cases, $2 \beta=\alpha-\gamma-2, \varepsilon \geqslant \frac{\beta}{4 A^{2(\alpha-1)} \text {, and }}$ $2 \beta<\alpha-\gamma-2$, bounded travelling-wave solutions do not exist. These results correspond to the formal asymptotic analysis in Hassanizadeh \& Gray [14].

It is worth observing that the limits $\varepsilon \rightarrow 0$ and $\delta \rightarrow 0$ do not commute. We can always take the limit $\varepsilon \rightarrow 0$ followed by $\delta \rightarrow 0$. However, the reverse is only possible if $2 \beta \geqslant \alpha-\gamma-2$.

Remark 1.1 Fixing $\delta \in(0, A)$, the existence is demonstrated for $\alpha>1$ and $\beta, \gamma>0$. All other results require in addition $\beta<\alpha-1$.

\section{Existence, uniqueness and monotonicity}

The main result of this section is:

Theorem 2.1 Let $\alpha>1, \beta, \gamma, \varepsilon>0$ and $0<\delta<A$. Further, let $c$ be given by (1.18). Then there exists a $C^{\infty}$ solution of Problem $T W$, unique up to translations in $\eta$. 
Proof We transform equation (1.17) into a planar system and apply a phase plane analysis. First we set $u=f^{1+\gamma}$, which gives

$$
c u^{\frac{1-\alpha}{1+\gamma}}-1=\left(c \delta-\delta^{\alpha}\right) u^{-\frac{\alpha}{1+\gamma}}+\frac{\beta}{1+\gamma} u^{\left(-\frac{\beta}{1+\gamma}-1\right)} u^{\prime}+\frac{\varepsilon c}{1+\gamma} u^{\prime \prime} \quad \text { on } \quad \mathbb{R}
$$

with boundary conditions

$$
u(-\infty)=\delta^{1+i}, \quad u(\infty)=A^{1+i}
$$

Next we put this equation in the Liénard phase-plane, by considering $u$ and $v:=$ $\frac{\varepsilon c}{1+\gamma} u^{\prime}-u^{-\frac{\beta}{1+\gamma}}$, as independent variables. This results in the system

$$
(P)\left\{\begin{array}{c}
\varepsilon u^{\prime}=F(u, v)=\frac{1+\gamma}{c}\left(v+u^{-\frac{\beta}{1+y}}\right) \\
v^{\prime}=G(u)=-1+c u^{\frac{1-x}{1+\gamma}}-\left(c \delta-\delta^{x}\right) u^{-\frac{x}{1+i}} .
\end{array}\right.
$$

A solution $f$ of $(T W)$ is an integral curve of $(P)$ connecting the equilibria $p_{--}=$ $\left(\delta^{1+\gamma},-\delta^{-\beta}\right)$ and $p_{+}=\left(A^{1+\gamma},-A^{-\beta}\right)$. The phase plane, with the isoclines $\Gamma_{u}=\{(u, v)$ : $F(u, v)=0\}$ and $\Gamma_{v}=\{(u, v): G(u)=0\}$, is drawn in Fig. 1.

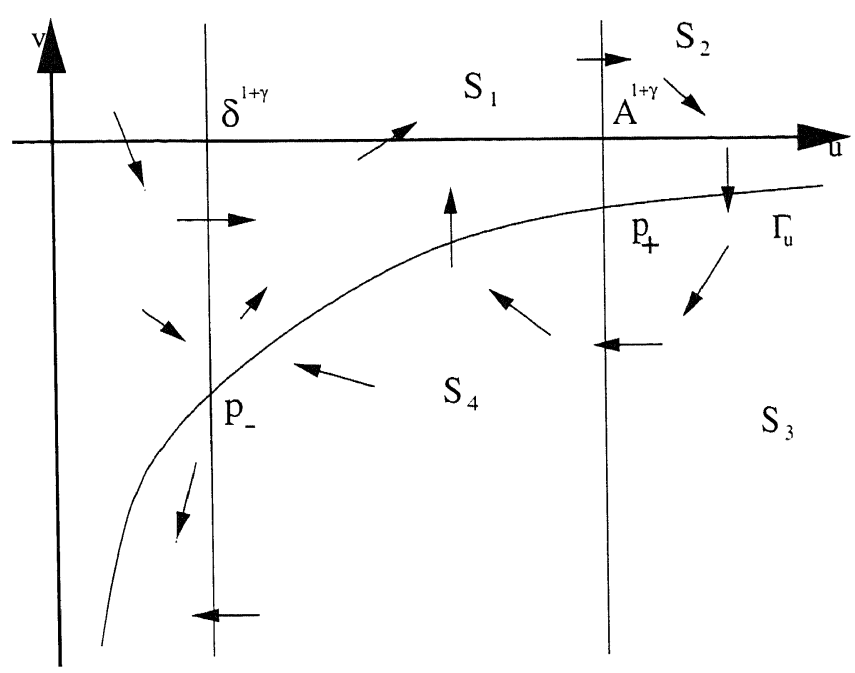

FIGURE 1. Phase plane for $(P)$.

The matrix of the linearised system is

$$
\left(\begin{array}{cc}
-\frac{\beta}{\varepsilon c} X^{-\beta-1-\gamma} & \frac{1+\gamma}{\varepsilon c} \\
\frac{X^{-\alpha-i-1}}{1+i}\left(c(1-\alpha) X+\left(c X-X^{\alpha}\right) \alpha\right) & 0
\end{array}\right),
$$

where $X=\delta$ corresponds to the equilibrium $p_{-}$, and $X=A$ to $p_{+}$. The eigenvalues in $p_{-}$ and $p_{+}$are, expressed in terms of $X$,

$$
\lambda=-a \pm \sqrt{a^{2}+b} \text { with } a=\frac{\beta}{2 \varepsilon c} \frac{1}{X^{\beta+\gamma+1}} \text { and } b=\frac{c-\alpha X^{\alpha-1}}{X^{\alpha+\gamma} \varepsilon c} .
$$


It is clear that $a>0$ for positive $X$, and $b$ has the sign of $c-X^{\alpha-1} \alpha$. Introducing its primitive

$$
i(X)=c X-X^{\alpha}
$$

we note that $A$ and $\delta$ are related by $i(A)=i(\delta)>0$, so that $b>0$ in $X=\delta$ and $b<0$ in $X=A$. Therefore, at $p_{-}$the two eigenvalues are real and of opposite sign, whence $p_{-}$is a saddle point. The point $p_{+}$is a sink and

$$
\begin{aligned}
& 0 \leqslant a^{2}+b<a^{2} \text { implies two real eigenvalues, } \lambda_{1} \leqslant \lambda_{2}<0 \text {, and } \\
& a^{2}+b<0 \text { implies two complex eigenvalues, with negative real part. }
\end{aligned}
$$

To prove Theorem 2.1 we have to show that an orbit leaving $p_{-}$connects to $p_{+}$. Because $p_{-}$is a saddle point there exist locally two orbits containing solutions $(u(\eta), v(\eta))$ of $(P)$, satisfying

$$
\lim _{\eta \rightarrow-\infty}(u(\eta), v(\eta))=p_{-} .
$$

Let $C$ be the orbit for which $u^{\prime}, v^{\prime}>0$. Inspection of Figure 1 shows that the other orbit will never reach $p_{+}$. The only possibility giving existence of a travelling wave is for $C$ to end up in $p_{+}$. The corresponding solutions will satisfy $(T W)$, and uniqueness up to translations in $\eta$ will hold.

Introducing the sets (see also Figure 1)

$$
\begin{aligned}
& S_{1}=\left\{(u, v) \in \mathbb{R}^{2}: \delta^{\gamma+1}<u<A^{\gamma+1}, v>-u^{-\frac{\beta}{1+\gamma}}\right\}, \\
& S_{2}=\left\{(u, v) \in \mathbb{R}^{2}: u>A^{\gamma+1}, v>-u^{-\frac{\beta}{1+\gamma}}\right\}, \\
& S_{3}=\left\{(u, v) \in \mathbb{R}^{2}: u>A^{\gamma+1}, v<u^{-\frac{\beta}{1+\gamma}}\right\}, \\
& S_{4}=\left\{(u, v) \in \mathbb{R}^{2}: \delta^{\gamma+1}<u<A^{\gamma+1}, v<-u^{-\frac{\beta}{1+\gamma}}\right\} .
\end{aligned}
$$

we have by straightforward phase plane arguments that either the orbit $C$ rotates around $p_{+}$, going from $S_{i}$ to $S_{i+1 \bmod 4}$, or it enters $p_{+}$from $S_{1}$ or $S_{3}$. Note that

$$
\operatorname{div}\left(\frac{1}{\varepsilon} F(u, v), G(u)\right)=-\frac{\beta}{c \varepsilon} u^{-\frac{\beta+\gamma+1}{\gamma+1}}<0 \quad \text { for all } u>\delta^{\gamma+1},
$$

so it is not possible for $C$ to connect back to $p_{-}$, or to exit $S_{4}$ through $u=\delta^{1+\gamma}$.

To complete the proof suppose that $(u(\eta), v(\eta))$ does not tend to $p_{+}$as $\eta \rightarrow \infty$. It then follows from arguments similar to the proof of Poincaré-Bendixon theorem that $C$ approaches a periodic orbit, again contradicting (2.5).

Next we derive a sufficient condition for the travelling-wave solution to have a monotone profile. This condition is related to the value of $\varepsilon$. Therefore we write $C=C_{\varepsilon}$ and $P=P_{\varepsilon}$ whenever appropriate.

From (2.2) we see that for all $\varepsilon$ such that

$$
\varepsilon<\varepsilon^{*}:=\frac{\beta^{2}}{4 c\left(A^{\alpha-1} \alpha-c\right)} A^{\alpha-2 \beta-\gamma-2} \quad\left(\varepsilon^{*}>0\right),
$$

the eigenvalues $\lambda_{1}, \lambda_{2}$ are real and strictly negative, which is a necessary condition for the travelling wave profile to be monotone. Henceforth, we suppose we are in this situation. With $\lambda_{2}<\lambda_{1}<0$ we call $\left(u_{1}, v_{1}\right)$ the slow eigenvector and $\left(u_{2}, v_{2}\right)$ the fast eigenvector at 
$p_{+}$, where

$$
\left(u_{1,2}, v_{1,2}\right)=\left(1, \frac{1}{2(1+\gamma)} \frac{\beta \pm \sqrt{\beta^{2}+4\left(c A-A^{\alpha} \alpha\right) \varepsilon c A^{2 \beta-\alpha+\gamma+1}}}{A^{1+\gamma+\beta}}\right)
$$

By standard local analysis (e.g. see Coddington \& Levinson [7]), there exist exactly two orbits entering $p_{+}$tangent to the $\left(u_{2}, v_{2}\right)$-direction: one along $\left(u_{2}, v_{2}\right)$, the other along $\left(-u_{2},-v_{2}\right)$. The connecting orbit goes around $p_{+}$at most a finite number of times.

Proposition 2.1 Let (1.13) be satisfied. For $\delta>0$ fixed, there exists $0<\bar{\varepsilon}<\varepsilon^{*}$, such that for every $0<\varepsilon<\bar{\varepsilon}$ the travelling wave obtained in Theorem 2.1 is strictly increasing on $\mathbb{R}$.

Proposition 2.1 is a direct consequence of Lemma 2.1 below, in which we construct an invariant region which contains $C$ and which itself is contained in $S_{1}$.

More specifically, for fixed $\delta \in(0, A)$ and $\mu \in(0,1)$, let $S_{\mu}^{\delta}$ denote the set enclosed by the curves

$$
u=\delta^{1+\gamma}, v=-u^{-\frac{\beta}{1+\gamma}} \quad \text { and } \quad v=g_{\mu}(u):=\left(-\mu u^{-\frac{\beta}{1+\gamma}}-(1-\mu) A^{-\beta}\right) .
$$

We will show in Proposition 2.1 that for $\varepsilon$ sufficiently small (i.e. $\varepsilon<\varepsilon_{\mu}^{\delta}$ ), $S_{\mu}^{\delta}$ is invariant for Problem $P_{\varepsilon}$ or, equivalently, $C_{\varepsilon} \in S_{\mu}^{\dot{\delta}}$. Proposition 2.1 will also be helpful in the study of the limits $\varepsilon \rightarrow 0$ and $\delta \rightarrow 0$. In particular, we want to bound $\varepsilon_{\mu}^{\delta}$ away from zero as $\delta \rightarrow 0$. It will appear that this is only possible if $2 \beta+\gamma+2-\alpha \geqslant 0$.

Lemma 2.1 For any fixed $\delta$ in $(0, A)$ and $\mu \in(0,1)$ there exists $\varepsilon_{\mu}^{\delta} \in\left(0, \varepsilon^{*}\right)$, such that for every $\varepsilon \in\left(0, \varepsilon_{\mu}^{\delta}\right), C_{\varepsilon} \in S_{\mu}^{\delta}$. Further, if $2 \beta+\gamma+2-\alpha \geqslant 0$,

$$
\lim _{\delta \rightarrow 0} \varepsilon_{\mu}^{\delta}=4 \mu(1-\mu) \lim _{\delta \rightarrow 0} \varepsilon^{*}=\mu(1-\mu) \frac{\beta^{2}}{4(\alpha-1)} A^{-2 \beta-\gamma-1},
$$

and

$$
\lim _{\delta \rightarrow 0} S_{\mu}^{\delta} \quad \text { is invariant for Problem } T W \text { with } \quad \delta=0
$$

Proof Observe that the eigenvectors at $p_{+}$satisfy

$$
\left(u_{1}, v_{1}\right) \rightarrow\left(1, \frac{\beta}{(1+\gamma) A^{1+\gamma+\beta}}\right), \text { and }\left(u_{2}, v_{2}\right) \rightarrow(1,0) \text { as } \varepsilon \rightarrow 0,
$$

where $\left(1, \frac{\beta}{(1+\gamma) A^{1+i+\beta}}\right)$ is the tangent vector at $p_{+}$to $\Gamma_{u}$. The invariant region $S_{\mu}^{\delta}$ is below the horizontal line $v=-A^{-\beta}$, and only contains orbits entering $p_{+}$along the slow eigenvector $\left(u_{1}, v_{1}\right)$. Observe that $g_{\mu}(u)>-u^{-\frac{\beta}{1+\gamma}}$ for all $0<u<A^{1+\gamma}$, and $g_{\mu}\left(A^{1+\gamma}\right)=-A^{-\beta}$. Obviously, the vector field is pointing inwards at boundary points of $S_{\mu}^{\delta}$ on $u=\delta^{1+\gamma}$ and $\Gamma_{u}$. It remains to examine the vector field on $v=g_{\mu}(u)$. We clearly must have

$$
\frac{d v}{d u}=\frac{\varepsilon c}{1+\gamma} \frac{G(u)}{g_{\mu}(u)+u^{-\frac{\beta}{1+\gamma}}} \leqslant g_{\mu}^{\prime}(u) \text { for all } u \in\left(\delta^{1+\gamma}, A^{1+\gamma}\right),
$$

for $S_{\mu}^{\delta}$ to be invariant. This is equivalent to

$$
\varepsilon H_{\delta}(u) \leqslant \mu(1-\mu)
$$


where

$$
H_{\delta}(u)=\frac{c}{\beta} \frac{-1+c u^{\frac{1-\alpha}{1+i}}-\left(c \delta-\delta^{\alpha}\right) u^{-\frac{\alpha}{1+\gamma}}}{\left(u^{-\frac{\beta}{1+\gamma}}-A^{-\beta}\right)} u^{1+\frac{\beta}{1+\gamma}} .
$$

Note that $H_{\delta}\left(\delta^{1+i}\right)=0$ and, by L'Hôpital's rule,

$$
\begin{aligned}
H_{\delta}\left(A^{1+\gamma}\right)=\lim _{u \rightarrow A^{1+\gamma}} & H_{\delta}(u)=c \frac{\alpha A^{\alpha-1}-c}{\beta^{2}} A^{2 \beta+\gamma+2-\alpha} \\
\rightarrow & \frac{(\alpha-1)}{\beta^{2}} A^{2 \beta+\gamma+\alpha} \text { as } \delta \rightarrow 0 .
\end{aligned}
$$

For $u>0$ fixed, $\lim _{\delta \rightarrow 0} H_{\delta}(u)$ behaves as $u^{\frac{2 \beta+++2-x}{1+y}}$ near 0 , which suggests we write $H_{\delta}(u)$ as

$$
\begin{array}{r}
H_{\delta}(u)=\frac{c}{\beta} u^{\frac{2 \beta+\gamma+2-\alpha}{1+\gamma}} h_{\delta}(u) \\
\text { where } h_{\delta}(u)=\frac{-u^{\frac{\chi}{1+\gamma}}+c u^{\frac{1}{1+\gamma}}-\left(c \delta-\delta^{\alpha}\right)}{u^{\frac{1}{1+\gamma}}\left(1-A^{-\beta} u^{\frac{\beta}{1+\gamma}}\right)}
\end{array}
$$

Observe that, for every $u \in\left(0, A^{1+\gamma}\right)$ and every $\delta>0$, recalling (1.18),

$$
h_{0}(u)-h_{\delta}(u)=\frac{\left(c \delta-\delta^{\alpha}\right)-u^{\frac{1}{1+\gamma}}\left(c-A^{\alpha-1}\right)}{u^{\frac{1}{1+\gamma}}\left(1-A^{-\beta} u^{\frac{\beta}{1+i}}\right)}>0,
$$

so that

$$
h_{\delta}(u)<h_{0}(u)=\frac{-u^{\frac{\alpha}{1+\gamma}}+A^{\alpha-1} u^{\frac{1}{1+\gamma}}}{u^{\frac{1}{1+\not}}\left(1-A^{-\beta} u^{\frac{\beta}{1+\gamma}}\right)}=A^{\alpha-1} \frac{1-A^{1-\alpha} u^{\frac{\alpha-1}{1+\gamma}}}{1-A^{-\beta} u^{\frac{\beta}{1+\gamma}}},
$$

which is increasing for $u \in\left(0, A^{1+\gamma}\right)$. Here we used $\beta<\alpha-1$ from (1.13).

Setting

$$
M:=\sup _{u \in\left(0, A^{1+i}\right)} h_{0}(u)=\frac{(\alpha-1) A^{\alpha-1}}{\beta},
$$

an upper bound for $H_{\delta}(u)$ is given by

$$
H_{\delta}(u)<M \frac{c}{\beta} A^{2 \beta+\gamma+2-\alpha} \quad \text { if } 2 \beta+\gamma+2-\alpha \geqslant 0,
$$

and

$$
H_{\delta}(u)<M \frac{c}{\beta} \delta^{2 \beta+\gamma+2-\alpha} \text { if } 2 \beta+\gamma+2-\alpha \leqslant 0 .
$$

Thus, a sufficient condition for (2.8) to hold is

$$
\varepsilon<\varepsilon_{\mu}^{\delta}:= \begin{cases}\mu(1-\mu) \frac{\beta^{2}}{c(\alpha-1)} A^{-2 \beta-\gamma-1} \quad \text { if } \quad 2 \beta+\gamma+2-\alpha \geqslant 0 \\ \mu(1-\mu) \frac{\beta^{2}}{c(\alpha-1) A^{\alpha-1}} \delta^{\alpha-2 \beta-\gamma-2} & \text { if } 2 \beta+\gamma+2-\alpha \leqslant 0 .\end{cases}
$$

This completes the proof of the first statement. The statements about the $\delta \rightarrow 0$ limit follow immediately from (2.13) with $2 \beta \geqslant \alpha-\gamma-2,(2.6)$ and (1.18). 


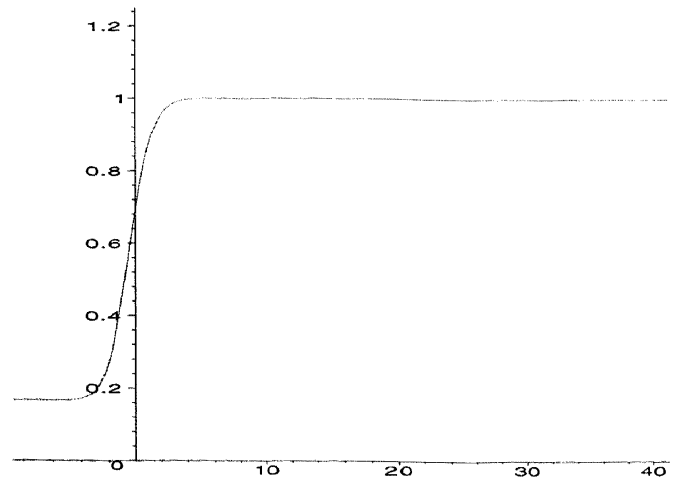

(a) Monotone profile: $\varepsilon=\varepsilon^{*}-0.01 \approx 0.048$.

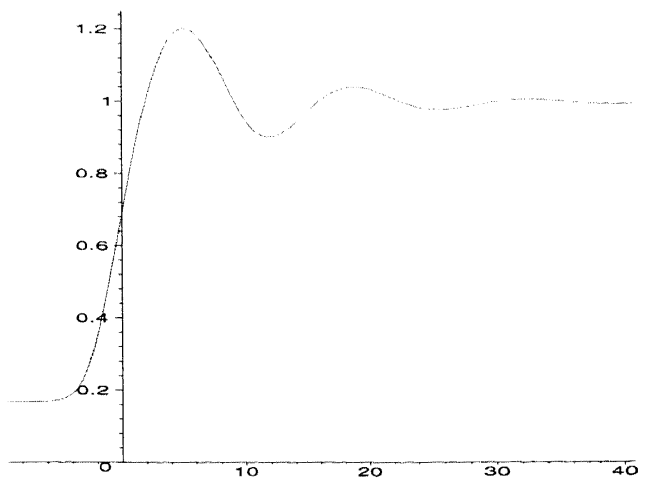

(b) Oscillatory profile: $\varepsilon=1$.

FIGURE 2. Travelling-wave solutions for different values of $\varepsilon$, where $f(0)=\frac{A+\delta}{2}, \alpha=\frac{4}{3}, \beta=\frac{1}{4}$, $\gamma=\frac{1}{2}, A=1, \delta=\frac{1}{6}$.

\section{The $\varepsilon \rightarrow 0$ limit case}

Let $\delta \in(0, A)$ be fixed and (1.13) be satisfied. In this section we examine the behaviour of the connecting orbit $C_{\varepsilon}$ and that of the corresponding travelling wave $f=f_{\varepsilon}$ as $\varepsilon \rightarrow 0$. For $\varepsilon<\bar{\varepsilon}$ we denote $C_{\varepsilon}$ by

$$
v=\varphi_{\varepsilon}(u), \quad \delta^{\gamma+1} \leqslant u \leqslant A^{\gamma+1}
$$

As a first convergence result we have

Proposition $3.1 \varphi_{\varepsilon}(u) \rightarrow-u^{-\frac{\beta}{1+i}}$ uniformly on $\left[\delta^{1+\gamma}, A^{1+\gamma}\right]$ as $\varepsilon \rightarrow 0$.

Proof Lemma 2.1 implies

$$
-u^{\frac{-\beta}{1+\gamma}}<\varphi_{\varepsilon}(u)<g_{\mu}(u)
$$

for all $u \in\left(\delta^{1+\gamma}, A^{1+\gamma}\right)$ and for all $\varepsilon \in\left(0, \varepsilon_{\mu}^{\delta}\right)$. Since $g_{\mu}(u) \rightarrow-u^{-\frac{\beta}{1+\gamma}}$ as $\mu \uparrow 1$, the result is immediate.

For the travelling waves $f_{\varepsilon}$ we have

Theorem 3.1 Translate $f_{\varepsilon}$ so that $f_{\varepsilon}(0)=\frac{\delta+A}{2}$ for all $\varepsilon>0$. Then $f_{\varepsilon} \rightarrow f \in C^{\infty}(\mathbb{R})$ as $\varepsilon \rightarrow 0$, uniformly on $\mathbb{R}$, where $f$ satisfies Problem $T W$ with $\varepsilon=0$.

Proof First we employ the scaling $\eta=\varepsilon \tau$, so that in the $\tau$-variable $\left(P_{\varepsilon}\right)$ reads

$$
\left(\tilde{P}_{\varepsilon}\right)\left\{\begin{array}{l}
\dot{u}=F(u, v), \\
\dot{v}=\varepsilon G(u) .
\end{array}\right.
$$


Unlike $\left(P_{0}\right)$, the limit system $\left(\tilde{P}_{0}\right)$, is well defined. The one-dimensional manifold of critical points

$$
M_{0}=\{F(v, u)=0\}=\left\{v=-u^{\frac{-\beta}{1+\gamma}}\right\}
$$

is invariant and normally hyperbolic in the sense of geometric singular perturbation theory (see Smoller [16]), because for $\left(\tilde{P}_{0}\right)$ the only pure imaginary eigenvalue is zero, and has a one-dimensional eigenspace tangential to $M_{0}$. Let $K$ be a neighbourhood of $\left\{(u, v) \in M_{0}: \delta^{1+\gamma} \leqslant u \leqslant A^{1+\gamma}\right\}$, and choose $0<\delta_{1}<\delta^{1+\gamma}<A^{1+\gamma}<A_{1}$ such that $\left\{(u, v) \in M_{0}: \delta_{1} \leqslant u \leqslant A_{1}\right\} \subset \subset K$. By Fenichel's invariant manifold theorem [16], there exists, for given $k \in \mathbb{N}$, a number $\varepsilon_{0}>0$ and a function $h \in C^{k}\left(\left[\delta_{1}, A_{1}\right] \times\left[0, \varepsilon_{0}\right]\right)$ with $h(u, 0)=-u^{-\frac{\beta}{1+\gamma}}$, such that for every $0<\varepsilon<\varepsilon_{0}$

$$
M_{\varepsilon}=\left\{(u, v) \in K: v=h(u, \varepsilon), \delta_{1} \leqslant u \leqslant A_{1}\right\}
$$

is locally invariant. The manifold $M_{\varepsilon}$ is not uniquely determined. However, between $u=\delta^{1+\gamma}$ and $u=A^{1+\gamma}$ it must coincide with the connecting orbit $v=\varphi_{\varepsilon}(u)$, because this is the only orbit which remains close to $\left\{v=-u^{-\frac{\beta}{1+\gamma}}: \delta_{1}<u<A_{1}\right\}$.

Using (3.1) and the $v$-equation in $\left(P_{\varepsilon}\right)$, we note that $u_{\varepsilon}=f_{\varepsilon}^{1+\gamma}$ satisfies,

$$
u^{\prime}=\frac{G(u)}{\varphi_{\varepsilon}^{\prime}(u)},
$$

and connects the two zeros of $G$.

Since $h \in C^{k}$ and $h(u, \varepsilon)=\varphi_{\varepsilon}(u)$ for $\delta^{1+\gamma} \leqslant u \leqslant A^{1+\gamma}$, we have as a result of Proposition 3.1, that $\varphi_{\varepsilon}^{\prime}(u) \rightarrow \frac{\beta}{1+\gamma} u^{-\frac{\beta}{1+\gamma}-1}$ uniformly on $\left[\delta^{1+\gamma}, A^{1+\gamma}\right]$, and thus

$$
\frac{G(u)}{\varphi_{\varepsilon}^{\prime}(u)} \rightarrow \frac{G(u)}{\frac{\beta}{1+\gamma} u^{-\frac{\beta}{1+\gamma}-1}}
$$

uniformly on $\left[\delta^{1+\gamma}, A^{1+\gamma}\right]$ as $\varepsilon \rightarrow 0$. In this limit, the differential equation (3.4) is identical to equation (2.1) with $\varepsilon=0$. Using the fact that $u_{\varepsilon}(0)$ is fixed for all $\varepsilon>0$, standard arguments imply that $u_{\varepsilon}$ converges uniformly on $\mathbb{R}$ to the corresponding solution of the limit equation.

\section{The $\delta=0$ system}

In this section we consider the limit case $\delta=0$ directly. Thus, we study the system

$$
\left(P_{\varepsilon}^{0}\right)\left\{\begin{array}{l}
\varepsilon u^{\prime}=F_{0}(u, v)=\frac{1+\gamma}{c}\left(v+u^{-\frac{\beta}{1+\gamma}}\right), \\
v^{\prime}=G_{0}(u)=-1+c u^{\frac{1-\gamma}{1+\gamma}},
\end{array}\right.
$$

where $c=A^{\alpha-1}$, and we look for orbits connecting $u=0$ to $u=A^{1+\gamma}$. The critical point corresponding to the latter now has real eigenvalues (see also (2.6)) for

$$
0<\varepsilon \leqslant \varepsilon^{*}=\frac{\beta^{2}}{4(\alpha-1)} A^{-\alpha-2 \beta-\gamma} .
$$

The phase plane (see Fig. 1) clearly implies that the desired orbit has to originate from the segment $\{(u, v): u=0, v \leqslant 0\}$ where the equations are singular. Since we are interested in $\left(P_{\varepsilon}^{0}\right)$ as limit of $\left(P_{\varepsilon}^{\delta}\right)$, and in particular of a possible limit orbit of the connecting orbit $C$, 
we expect such a limit orbit, if it exists, to behave as $v \sim-d u^{-\frac{\beta}{1+\gamma}}, 0<d \leqslant 1$, as $u \rightarrow 0$. Thus, a convenient new dependent variable is $Z=u^{q} v$, where $q$, for later purposes, is not fixed yet. Whenever $u^{\prime} \neq 0, Z$ satisfies the equation

$$
u \frac{d Z}{d u}=q Z+\frac{\varepsilon c}{1+\gamma} u^{1+2 q+\frac{1-x}{1+i}} \frac{c-u^{\frac{2-1}{1+\gamma}}}{Z+u^{q-\frac{\beta}{1+i}}} .
$$

Below we investigate the solvability of (4.1) for $0<u<A^{1+\gamma}$. The analysis and results critically depend on the value of the parameters $\alpha, \beta$ and $\gamma$. In particular, the value of $2 \beta-\alpha+\gamma+2$ plays a crucial role, which is to be expected considering the results of the formal analysis by Hulshof \& King [14]. With $q$ appropriately chosen, we consider the cases below.

$$
\text { 4.1 The case } 2 \beta>\alpha-\gamma-2
$$

Here we take $q=\frac{\beta}{1+\gamma}$, and set $W=u^{\frac{2 \beta+1+2-x}{1+\gamma}}$. Then (4.1) becomes

$$
(2 \beta+\gamma+2-\alpha) \frac{d Z}{d W}=\frac{\beta Z}{W}+\frac{\varepsilon c\left(c-W^{\frac{z-1}{2 \beta+\gamma+2-\alpha}}\right)}{Z+1} .
$$

We look for solutions of (4.2) with $Z>-1$ as $W \rightarrow 0$ (i.e. $u^{\prime}>0$ as $u \rightarrow 0$ ). In Fig. 3 we sketch the $(W, Z)$-phase plane. Equation (4.2) and the phase plane imply that $Z \rightarrow Z_{0} \in\{0,-1\}$ as $W \rightarrow 0$, where orbits with $Z_{0}=0$ have $v=o\left(-u^{-\frac{\beta}{1+i}}\right)$, while orbits with $Z_{0}=-1$ have $v \sim-u^{\frac{\beta}{1+\gamma}}$.

Proposition 4.1 For $2 \beta>\alpha-\gamma-2$ there is a unique orbit $C^{0}$ with $u \rightarrow 0$ and $v \sim-u^{-\frac{\beta}{1+\gamma}}$ as $\eta$ decreases. This orbit reaches $(u, v)=(0,-\infty)$ at some finite $\eta$-value, implying the existence of a travelling wave with a front. The local behaviour of the front is determined by the relation

$$
f^{\prime} \sim \frac{c}{\beta} f^{\beta+2-\alpha} \text { as } f \rightarrow 0 .
$$

Proof We first prove existence. Choose $W_{0}$ small and denote the solution of (4.2) with $Z=\xi$ in $W=W_{0}$ by $Z=Z(W, \xi)$. Let

$$
\begin{aligned}
& S_{+}=\{\xi \in(-1,0): Z(W, \xi) \rightarrow 0 \text { as } W \downarrow 0\} \\
& S_{-}=\left\{\xi \in(-1,0): \exists W_{*} \in\left(0, W_{0}\right) Z(W, \xi) \rightarrow-1 \text { as } W \downarrow W_{*}>0\right\} \\
& S_{0}=\{\xi \in(-1,0): Z(W, \xi) \rightarrow-1 \text { as } W \downarrow 0\} .
\end{aligned}
$$

By standard arguments we have for $W_{0}$ sufficiently small that

$$
(0,-1)=S_{-} \cup S_{0} \cup S_{+}
$$

and $S_{-}$and $S_{+}$are nonempty and open. Hence $S_{0}$ is nonempty, which gives existence. We observe that for such an orbit (see (4.2)),

$$
Z+1 \sim a W \text { as } W \downarrow 0 \text { where } a=\frac{\varepsilon c^{2}}{\beta} .
$$

Next we prove uniqueness. Suppose there are two solutions $Z=Z_{1}(W)$ and $Z=Z_{2}(W)$ 
with $Z \rightarrow-1$ as $W \downarrow 0$. Since

$$
\frac{1}{Z_{1}+1}-\frac{1}{Z_{2}+1}=-\frac{1}{(\tilde{Z}+1)^{2}}\left(Z_{1}-Z_{2}\right)
$$

where $\tilde{Z}$ lies between $Z_{1}$ and $Z_{2}$, we have for $Y=Z_{1}-Z_{2}>0$, say,

$$
\frac{d Y}{d W}-b \frac{Y}{W} \sim-b^{\prime} \frac{Y}{W^{2}} \quad \text { as } W \rightarrow 0,
$$

where $b=\frac{\beta}{2 \beta+\gamma+2-\alpha}$, and $b^{\prime}=\frac{\beta}{\varepsilon c^{2}(2 \beta+\gamma+2-\alpha)}$. Here we used (4.3). Hence, $Y \rightarrow \infty$ as $W \downarrow 0$, contradicting (4.3).

Expressing $W$ and $Z$ in terms of $f$ we observe that (4.3) implies the behaviour

$$
f^{\prime} \sim \frac{c}{\beta} f^{\beta+2-\alpha} \text { as } f \rightarrow 0
$$

Since $\beta+2-\alpha<1$, we find that $f$ reaches zero at some finite $\eta$-value, i.e. the travelling wave has a finite front.

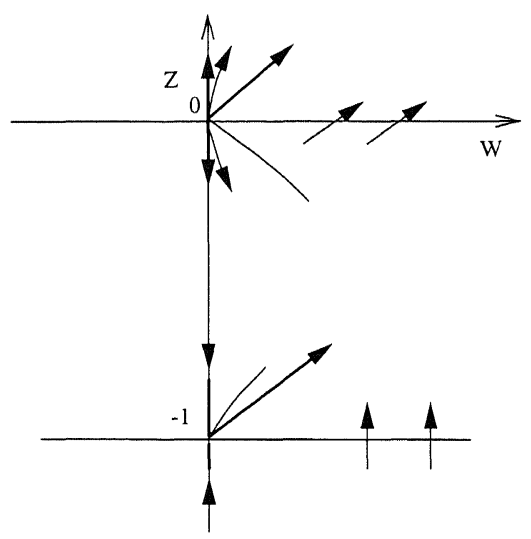

FIGURE 3. Phase plane related to (4.2), with $q=\frac{\beta}{1+\gamma}$.

Note that we did not put any restriction on $\varepsilon$. Thus the conclusion about the behaviour as $f \rightarrow 0$, provided $2 \beta>\alpha-\gamma-2$, is valid for any value of $\varepsilon \geqslant 0$. This $\varepsilon$-uniformity is lost in the next case.

Indeed, the behaviour of the travelling wave near the front when it vanishes corresponds to criterion (1.12) in the case where the capillary damping is absent.

4.2 The case $2 \beta=\alpha-\gamma-2$

Now we take $q=\frac{\beta}{1+\gamma}$, and set $W=u^{\frac{x-1}{1+\gamma}}$. Then (4.1) becomes

$$
(\alpha-1) \frac{d Z}{d W}=\left(\beta Z+\frac{\varepsilon c(c-W)}{(Z+1)}\right) \frac{1}{W} .
$$


gain, we look for solutions satisfying $Z>-1$ and $Z \rightarrow Z_{0}$ as $W \rightarrow 0$. It follows that

$$
Z_{0}^{ \pm}=-\frac{1}{2} \pm \frac{1}{2}\left(1-\frac{4 \varepsilon c^{2}}{\beta}\right)^{\frac{1}{2}}
$$

ıplying $\varepsilon \leqslant \frac{\beta}{4 c^{2}}$. With reference to Fig. 4 we have

oposition 4.2 (i) If $\varepsilon<\frac{\beta}{4 c^{2}}$, there exists a family of orbits satisfying $v \sim Z_{0}^{+} u^{-\frac{\beta}{1+i ̈}}$ as $u \rightarrow 0$, and a unique orbit, denoted by $C^{0}$, which satisfies $v \sim Z_{0}^{-} u^{-\frac{\beta}{1+\gamma}}$ as $u \rightarrow 0$. All cases give travelling waves with finite fronts. In particular, the orbit $C^{0}$ implies

$$
f^{\prime} \sim \frac{1}{\varepsilon c}\left(1-\left(1-\frac{4 \varepsilon c^{2}}{\beta}\right)^{\frac{1}{2}}\right) f^{\beta+2-\alpha}, \quad \text { as } f \rightarrow 0 .
$$

(ii) If $\varepsilon=\frac{\beta}{4 c^{2}}$ there exists a family of orbits having $v \sim-\frac{1}{2} u^{-\frac{\beta}{1+\gamma}}$ as $u \rightarrow 0$. In particular, there is a unique orbit, again denoted by $C^{0}$, satisfying $u^{\frac{\beta}{1+7}} v \uparrow-\frac{1}{2}$ as $u \rightarrow 0$. The orbit $C^{0}$ implies again a travelling wave with a finite front, such that

$$
f^{\prime} \sim \frac{2 c}{\beta} f^{\beta+2-\alpha}, \quad \text { as } f \rightarrow 0 .
$$

(iii) If $\varepsilon>\frac{\beta}{4 c^{2}}$, there is no orbit with $v>-u^{\frac{-\beta}{1+i}}$ and $u \rightarrow 0$.

emark 4.1 (i) Comparing (2.6) and (2.13), we observe that

$$
\varepsilon_{\frac{1}{2}}^{0}=\varepsilon^{*}=\frac{\beta^{2}}{4(\alpha-1) c^{2}}<\frac{\beta}{4 c^{2}},
$$

implying that, depending on the $\varepsilon$-value, monotone and oscillatory waves with finite fronts occur.

(ii) Since now $\beta+2-\alpha=-\beta-\gamma<0$, finite front waves have $f^{\prime} \rightarrow \infty$ as $f \rightarrow 0$.

\section{roof}

(i) It is immediate from the phase plane in Fig. 4 that $\left(0, Z_{0}^{+}\right)$is a source, and $\left(0, Z_{0}^{-}\right)$ is a saddle, with one unique orbit $Z=Z(W)$ leaving in the direction $W>0$. It behaves as $Z-Z_{0}^{-} \sim\left(\frac{1}{2}-\frac{1}{2}\left(1-\frac{4 \varepsilon c^{2}}{\beta}\right)^{\frac{1}{2}}\right)$ as $W \rightarrow 0$.

(ii) Now a local analysis near $Z_{0}=-\frac{1}{2}$ shows that $\left(0, Z_{0}\right)$ is a saddle-node, with a unique orbit in the direction of $W>0$. This orbit does not cross the isocline, and behaves as $Z-Z_{0} \sim \frac{1}{2} W$.

(iii) Now the segment $\{(W, Z): W=0, Z \in(-1,0)\}$ is disconnected from the isocline, and hence no connecting orbit exists. 

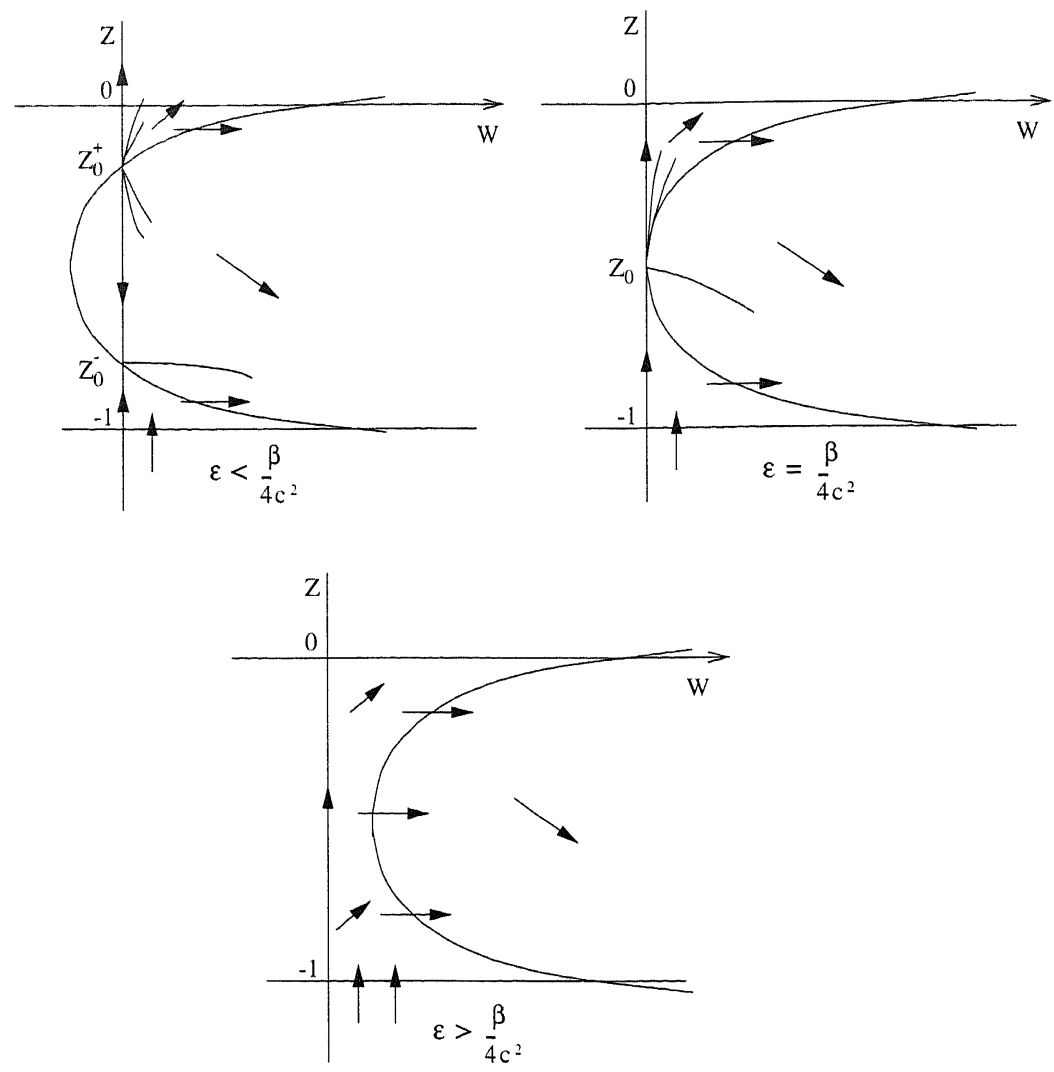

FIGURE 4. $q=\frac{\beta}{1+\gamma}, 2 \beta=\alpha-\gamma-2$ phase plane for different $\varepsilon$-values.

4.3 The case $2 \beta<\alpha-\gamma-2$

Here we choose $q=\frac{\alpha-2-\gamma}{2(1+\gamma)}>\frac{\beta}{1+\gamma}$ and $W=u^{q-\frac{\beta}{1+\gamma}}$, yielding the equation

$$
(\alpha-\gamma-2-2 \beta) \frac{d Z}{d W}=(\alpha-\gamma-2) \frac{Z}{W}+\frac{2 \varepsilon c\left(c-W^{\frac{2(\alpha-1)}{\alpha-i-2-2 \beta}}\right)}{(W+Z) W}
$$

Proposition 4.3 There exists no orbits with $v>-u^{-\frac{\beta}{1+\gamma}}$ and $u \rightarrow 0$.

Proof Suppose such an orbit exists. Then we would have

$$
c(\alpha-\gamma-2-2 \beta) \frac{d Z}{d W} \sim \frac{1}{W}\left((\alpha-\gamma-2) Z+\frac{2 \varepsilon c^{2}}{Z}\right), Z>0,
$$

as $W \downarrow 0$. Since $(\alpha-\gamma-2) Z+\frac{2 \varepsilon c^{2}}{Z}$ is negative and bounded away from zero, this gives contradiction. 
5 The limit $\delta \rightarrow 0$

For the purpose of this section, we denote the connecting orbit of $\left(P_{\varepsilon}^{\delta}\right)$ by $v=\varphi_{\delta}(u)$ and, in the cases for which $C^{0}$ from Proposition 4.1 and 4.2 exists, we call its graph $v=\varphi_{0}(u)$.

Lemma 5.1 There exists $\delta^{*}>0$ such that, with $c=c(\delta)=\frac{A^{x}-\delta^{x}}{A-\delta}$,

$$
\mathscr{F}_{u}(\delta)=c(\delta) G_{\delta}(u)=c(\delta)\left(-1+c(\delta) u^{\frac{1-\chi}{1+j}}-c(\delta) A-A^{\alpha} u^{-\frac{z}{1+\gamma}}\right)
$$

is decreasing in $0 \leqslant \delta<\delta^{*}$ for any fixed $0<u \leqslant\left(\frac{A}{2}\right)^{1+\gamma}$.

Proof Since

$$
\frac{d \mathscr{F}_{u}}{d \delta}=\frac{d c}{d \delta}\left(-1+2 c u^{\frac{1-\chi}{1+\gamma}}+\left(A^{\alpha}-2 c A\right) u^{-\frac{\chi}{1+\gamma}}\right),
$$

and $\frac{d c}{d \delta}>0$, we need to show that the term in large brackets is negative for small $\delta$. At $\delta=0$ it becomes

for all $0<u<\left(\frac{A}{2}\right)^{1+\gamma}$.

$$
-1+A^{\alpha-1} u^{-\frac{\alpha}{1+\gamma}}\left(2 u^{\frac{1}{1+\gamma}}-A\right)<0,
$$

Proposition 5.1 For $\alpha, \beta, \gamma$ such that $2 \beta>\alpha-\gamma-2$, or $2 \beta=\alpha-\gamma-2$ and $\varepsilon \in\left(0, \frac{\beta}{4 c^{2}}\right)$ fixed, translate $f_{\delta}$ such that $f_{\delta}(0)=\frac{A}{2}$ for all $\delta \in\left(0, \delta^{*}\right)$. Then $f_{\delta} \rightarrow f \in C^{\infty}(\mathbb{R})$ uniformly on $\mathbb{R}$. Hence $f$ satisfies Problem $T W$ with $\delta=0$.

Proof It will be sufficient to show that $\varphi_{\delta}(u) \rightarrow \varphi_{0}(u)$ locally uniformly. By Lemma 5.1 we have for any $0<\bar{\delta} \leqslant \delta^{*}$

$$
-u^{-\frac{\beta}{1+\gamma}}<\varphi_{\delta_{1}}(u)<\varphi_{\delta_{2}}(u)<\varphi_{0}(u)
$$

for $0<\delta_{1}<\delta_{2}<\delta^{*}$ and $u \in\left(\bar{\delta},\left(\frac{A}{2}\right)^{1+\gamma}\right)$. Also

$$
\frac{\varepsilon c(\delta)}{1+\gamma} \frac{G_{\delta}(u)}{F_{\delta}(u, v)} \rightarrow \frac{\varepsilon A^{\alpha-1}}{1+\gamma} \frac{G_{0}(u)}{F_{0}(u, v)} \text { as } \delta \rightarrow 0
$$

uniformly on $\left[\bar{\delta}^{1+\gamma},\left(\frac{A}{2}\right)^{1+\gamma}\right]$. Therefore,

$$
\varphi_{\delta}(u) \uparrow \bar{\varphi}(u) \leqslant \varphi_{0}(u)
$$

where $v=\bar{\varphi}_{0}(u)$ is a solution of $\left(P_{\varepsilon}^{0}\right)$. The reasoning above holds for every $0<\bar{\delta} \leqslant \delta^{*}$, which implies that $\bar{\varphi}_{0}(u)$ exists for all $u \in\left(0,\left(\frac{A}{2}\right)^{1+\gamma}\right)$. In view of $\S 4$ and $\bar{\varphi} \leqslant \varphi_{0}$, this implies that $\bar{\varphi}_{0}(u)=\varphi_{0}(u)$.

Using that $f_{\delta}(0)=\frac{A}{2}$ is fixed for all $0<\delta<\delta^{*}$, standard arguments imply that $f_{\delta}$ converges uniformly on $\left(-\infty, \frac{A}{2}\right)$ to the corresponding solution of the limit equation. Existence of global travelling waves and uniqueness of the initial value problem for all $0 \leqslant \delta<\delta^{*}$, implies uniform convergence on $\mathbb{R}$.

\section{Concluding remarks}

In this paper we present a study of a model for unsaturated groundwater flow which includes expression (1.4) for the non-static phase pressure difference. Replacing the non- 
linearities in the transport equation by power-law expressions we arrive at (1.10). We study travelling-wave solutions representing moisture profiles moving downwards due to gravity.

For positive initial saturation $(\delta>0)$ we demonstrate existence and uniqueness (up to translations). Small values of the damping coefficient $\varepsilon$ result in monotone saturation profiles. Large values of $\varepsilon$ result in profiles which exhibit oscillatory behaviour near the injection saturation $A$.

When initially no moisture is present $(\delta=0)$, the existence of bounded travelling waves depends critically upon the exponents of the power-law expressions. This is related to the occurrence of finite fronts in the moisture profiles, i.e. descending planes (in the direction of gravity) below which the water saturation remains zero. Related to equation (1.10) we have shown the following.

If $2 \beta>\alpha-\gamma-2$, then travelling-wave solutions with fronts exist for all $\varepsilon>0$. In other words, for $S(z, t)=f(\eta)$, with $\eta=z+c t$, there exists $\eta_{0} \in \mathbb{R}$ such that $f(\eta)=0$ for all $\eta \leqslant \eta_{0}$. Moreover, near $\eta=\eta_{0}$ the profile satisfies

$$
f^{\prime} \sim \frac{A^{\alpha-1}}{\beta} f^{\beta+2-\alpha} .
$$

This corresponds to the front behaviour of solutions of the convection diffusion equation under static conditions (equation (1.11)), i.e. $\varepsilon$ and $\gamma$ are absent in this asymptotic expression.

If $2 \beta=\alpha-\gamma-2$ we obtain a similar result provided the damping coefficient $\varepsilon$ is sufficiently small: i.e. $\varepsilon \leqslant \frac{\beta}{4 A^{2}(\alpha-1)}$. For larger values of $\varepsilon$, no waves exist satisfying $f(-\infty)=0$. Finally, if $2 \beta<\alpha-\gamma-2$, again no such waves exist, regardless the value of $\varepsilon>0$.

Let us interpret this in terms of the nonlinear functions, $K(S), p_{c}(S)$ and $L(S)$, as they were approximated in (1.7), (1.8) and (1.9). If we write the condition for existence of travelling-wave solution with fronts as $\gamma>\alpha-2(\beta+1)$, this implies that if $\alpha<2(\beta+1)$, then for any positive value of $\gamma$ fronts exist. But instead, if $\alpha>2(\beta+1)$, then $\gamma$ needs to be sufficiently large to have fronts solutions. In other words, if $K(S)$ is small near $S=0$ in the sense that $\alpha>2(\beta+1)$, then $L(S)$ must decay sufficiently fast near $S=0$, i.e. $\gamma>\alpha-2(\beta+1)$, to have fronts. We can express the condition for existence of travelling-wave solutions with fronts as

$$
L(S)=o\left(K(S) p_{c}^{\prime}(S)^{2}\right) \text { as } S \rightarrow 0 .
$$

We also investigate the limit $\varepsilon \rightarrow 0$ (for $\delta>0$, fixed) and $\delta \rightarrow 0$ (for $\varepsilon>0$ fixed). In particular, the latter provides a uniqueness criterion for the degenerate case when $\delta=0$. We also note that the limits $\varepsilon \rightarrow 0$ and $\delta \rightarrow 0$ do not commute: $\varepsilon \rightarrow 0$ followed by $\delta \rightarrow 0$ is always possible, while $\delta \rightarrow 0$ followed by $\varepsilon \rightarrow 0$ is only possible when $2 \beta \geqslant \alpha-\gamma-2$.

\section{References}

[1] Barenblatt G. I., García-Azorero J., De Pablo A. \& Vázquez J. L. (1997) Mathematical model of the non-equilibrium water-oil displacement in porous strata. Applicable Analysis, 65, 19-45. 
[2] Barenblatt, G. I., Entov, V. M. \& Ryzhik, V. M. (1990) Theory of Fluid Flow through Natural Rocks. Kluwer.

[3] Bear, J. (1988) Dynamics of Fluids in Porous Media. Dover.

[4] BEAR, J. (1979) Hydraulics of Groundwater. McGraw-Hill.

[5] Bedrikovetsky, P. (1993) Mathematical Theory of Oil and Gas Recovery. Kluwer.

[6] Bourgeat, A. \& Panfilov, M. (1998) Effective two-phase flow through highly heterogeneous porous media: Capillary nonequilibrium effects. Computational Geosciences, 2, 191-215.

[7] Coddington, E. \& Levinson, N. (1995) Theory of Ordinary Differential Equations. McGrawHill.

[8] Dibenedetto, E. \& Pierre, M. (1981) On the maximum principle for pseudoparabolic equations. Indiana Univ. Math. J., 30, 821-854.

[9] VAN Duijn, C. J. \& PeleTIER, L. A. (1982) Nonstationary filtration in partially saturated porous media. Archs. ratio. Mech. Analysis, 78, 173-198.

[10] Gilding, B. H. (1988) The occurrence of interfaces in nonlinear diffusion-advection processes. rch. Rational Mech. Anal. 100, 243-263.

[11] Hassanizadeh, S. M. May (1997) Dynamic Effects in the capillary pressure saturation relationship. Proceedings of 4th Int. Conf. on Civil Engineering, Tehran, Iran, 4, 141-149.

[12] Hassanizadeh, S. M. \& Gray, W. G. (1993) Thermodynamic basis of capillary pressure in porous media. Water Resources Research, 29, 3389-3405.

[13] Hulshof, J. (1987) An elliptic-parabolic free boundary problem: continuity of the free boundary. Proc. Roy. Soc. Edin. 106A, 327-339.

[14] Hulshof, J. \& King, J. R. (1998) Analysis of a Darcy flow model with a dynamic pressure saturation relation. SIAM J. Appl. Math. 59(1), 318-346.

[15] Smiles, D. E., Vachaud, G. \& Vauclin, M. (1971) A test of the uniqueness of the soil moisture characteristic during transient, non-hysteretic flow of water in rigid soil. Soil Sci. Soc. Am. Proc. 35, 535-539.

[16] Smoller, J. (1994) Shock Waves and Reaction-diffusion Equations (2nd ed). Springer-Verlag. 